Die Geschichte des Zürcher Medizinstudiums steht im Mittelpunkt des Sammelbands «Innenansichten einer Ärzteschmiede», der anlässlich des Universitätsjubiläums im Chronos-Verlag erschienen ist. Die Schweizerische Ärztezeitung druckt Auszüge aus den Artikeln in einer Serie ab. Unter dem Titel «Vom Grünschnabel zum Weisskittel» zeigt das Medizinhistorische Archiv zudem bis 31. Mai 2008 eine Ausstellung zum Thema im Hauptgebäude der Universität (www. 175jahre.uzh.ch/gruenschnabel-weisskittel).
Der Beitrag, aus dem die heutigen Auszüge stammen, beleuchtet die Debatten um Reformen des eidgenössischen Medizinstudiums zwischen 1877 und 1935, in denen sich allgemeine Vorstellungen über die Medizin spiegeln. Neben Fragen wie Studiendauer, Lehrfächer, Prüfungsordnungen und Praxisbezug der Ausbildung ging es in den 1920er Jahren auch um die Didaktik des Medizinstudiums.

\title{
Gelehrte oder Praktiker? Debatten über das ideale Medizinstudium*
}

\section{Eberhard Wolff}

\footnotetext{
* Auszug aus dem Beitrag des Autors in: Iris Ritzmann, Wiebke Schweer, Eberhard Wolff (Hrsg.). Innenansichten einer Ärzteschmiede. Lehren, lernen und leben - aus der Geschichte des Zürcher Medizinstudiums. Zürich: Chronos; 2008. 240 Seiten, 80 teils farbige Abbildungen. Fr. 34.-, € 20.60. ISBN 978-3-0340-0909-6. Quellenverweise im Originalartikel.
}

Korrespondenz:

Dr. rer. soc. Eberhard Wolff Universität Zürich

Medizinhistorisches Institut

Hirschengraben 82

CH-8001 Zürich

Tel. 0446342079

eberhard.wolff@mhiz.uzh.ch
Es war vor allem ein Arzt namens Berg aus Appenzell (wahrscheinlich handelte es sich um den aus Deutschland oder Österreich eingewanderten Victor Berg, 1884-1935), der in dieser Frage 1922 deutlich Stellung bezog. Berg stützte sich auf eigene Erfahrungen als Medizinstudent an der Universität Zürich sowie als Repetitor für 500 Studierende, mit denen er als bezahlter $\mathrm{Zu}$ satzlehrer den Stoff zur Prüfungsvorbereitung nochmals durchging. Bei dieser Tätigkeit habe er, Berg, Gelegenheit gehabt, «besser als irgend

\section{Soll ein Medizinstudium vor allem positivistisch Fakten vermitteln? Oder soll es helfen, Zusammenhänge zu verstehen, zu reflektieren und möglicher- weise zu kritisieren?}

ein Dozent oder Examinator feststellen zu können, was die Studenten wirklich in den Vorlesungen, Kursen und Kliniken profitiert hatten». Er habe die «Erfahrung gemacht, dass der Effekt, der geistige Gewinn insbesondere der Vorlesungen, aber auch der Kurse und Kliniken für den Studenten im allgemeinen ein auffallend geringer ist und dass das, was diese Studenten während des Semesters als sich rein rezeptiv verhaltende Zuhörer wirklich lernen, in gar keinem
Verhältnis steht zu der Zeit, welche in den Vorlesungen, Kursen und Kliniken erbracht [sic!] wird, auch in keinem Verhältnis zu der Energie, welche die meisten Dozenten in durchaus anzuerkennender Weise für die Belehrung ihrer Studenten aufwenden». Konkret schlug er vor: «Es soll doch einmal ein Dozent nach einem Jahr Vorlesung über Physiologie ein paar intelligente Studenten examinieren über das, was er ihnen ein Jahr lang vorgetragen hat. Er würde sicher erschrecken.» Den Grund für die miserable Bilanz sah er im Unterrichtsstil, der die Tätigkeit der Studierenden auf die reine Rezeption von Wissen reduziere. «Ich darf auf Grund vielfältiger Erfahrung füglich behaupten, dass das Nachschreiben, d.h. die Deponierung des Gehörten in möglichst vollständigen Kollegheften, also quasi das Schreiben nach Diktat, die geistige Haupttätigkeit der grossen Mehrzahl der Studenten darstellt und dass von den Dozenten viel mehr an das Gedächtnis der Studenten appelliert wird als an die geleistete produktive Mitarbeit.»

Berg schloss daraus, dass die Vernachlässigung didaktisch-pädagogischer Grundsätze einen «der schwersten Mängel unseres heutigen Studienplanes» darstelle. «Ganze Generationen von Medizinstudenten werden in verschiedenen Kollegien in unverantwortlicher Weise infolge der pädagogischen Unfähigkeit der betreffenden Dozenten gelangweilt; aber gewöhnlich ist es gerade diese Art von Dozenten, welche in schulmeisterlicher Weise den Vorlesungsbesuch kontrolliert und die Studenten ihre Examensmacht spüren lässt.» 


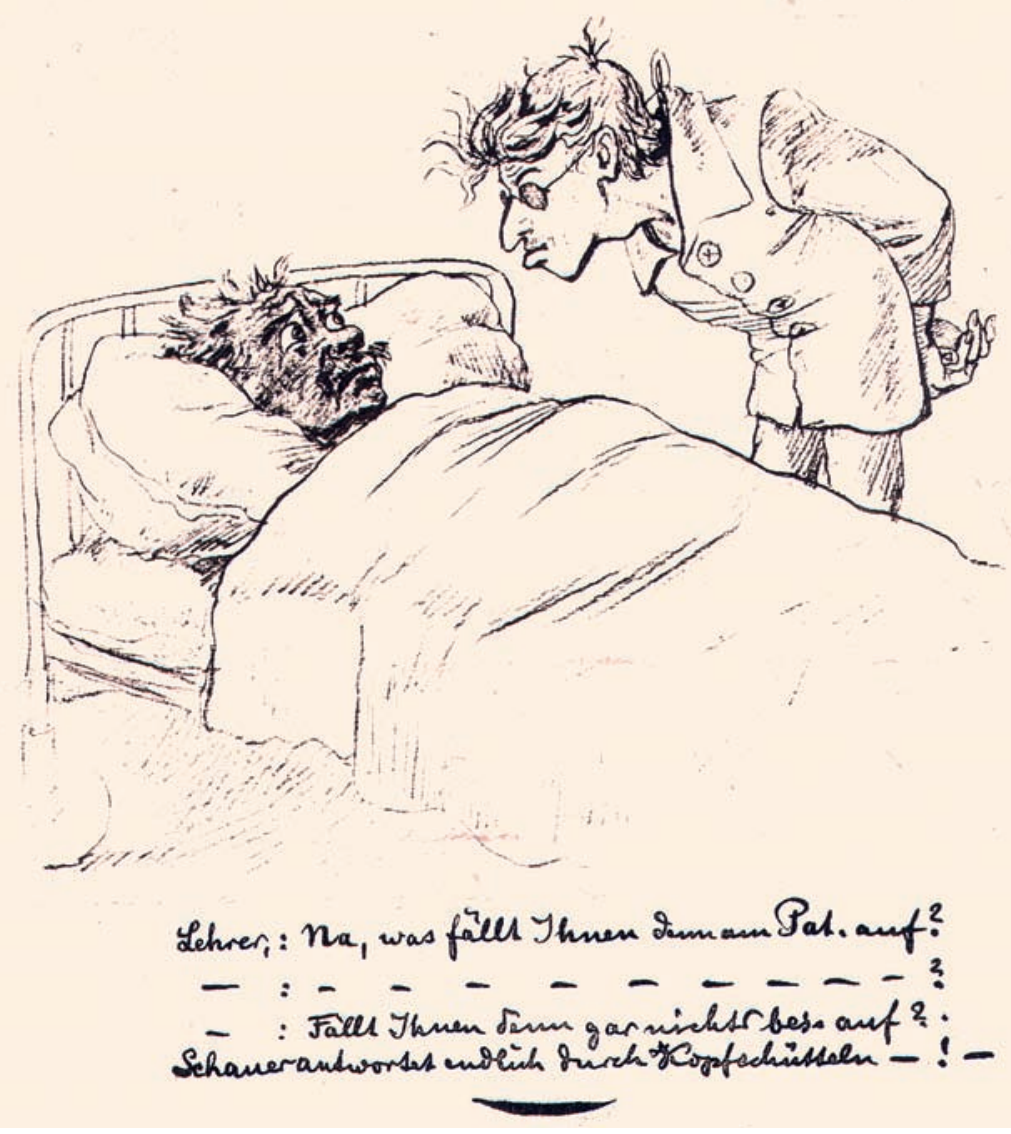

Prüfung am Krankenbett. Karikatur aus der Zürcher «Klinicisten Bier-Zeitung», Weihnachten 1894. (Medizinhistorisches Archiv, Universität Zürich)
Bergs Ausführungen zeigen mehr als den Gegensatz zwischen guter und schlechter Didaktik. Sein Hauptkritikpunkt trifft das Selbstverständnis der klassischen Ordinarienuniversität, deren Mittelpunkt die «Vorlesung» bildet, und dies im ganz konkreten Sinn des Wortes. Der Professor steht am Rednerpult und «liest» sein Fach vor. Die Aufgabe der Studenten ist es, diesen Stoff aufzunehmen und allenfalls zu Hause zu verarbeiten.

Bergs Kritik spricht indes noch eine weitere Dimension an. Soll ein Medizinstudium vor allem positivistisch Fakten vermitteln? Oder soll es helfen, Zusammenhänge zu verstehen, zu reflektieren und möglicherweise zu kritisieren? Das Zürcher Universitätsstudium war Bergs Meinung nach nicht auf letzteres ausgerichtet: «Ich kannte so manche geistig gar sehr beschränkte Kommilitonen, die alle Examina glatt absolvierten, weil sie über ein gutes Gedächtnis verfügten, ein geduldiges Sitzleder hatten und recht brave Studenten waren.»

In der nächsten Folge: Der «Hexenmeister» vom Burghölzli: Auguste Forels Hypnotismus im Vorlesungssaal und in der Klinik. 